\title{
Giant magnetoresistance ratio in a current-perpendicular-to-plane spin valve based on an inverse Heusler alloy $\mathrm{Ti}_{2} \mathrm{NiAl}$
}

\author{
Yu Feng ${ }^{1,2}$, Zhou Cui ${ }^{1}$, Bo $\mathrm{Wu}^{3}$, Jianwei $\mathrm{Li}^{1}$, Hongkuan Yuan ${ }^{2}$ and Hong Chen ${ }^{* 2}$
}

\author{
Full Research Paper \\ Address: \\ ${ }^{1}$ School of Physics and Electronic Engineering, Jiangsu Normal \\ University, Xuzhou 221116, People's Republic of China, ${ }^{2}$ School of \\ Physical Science and Technology, Southwest University, Chongqing \\ 400715, People's Republic of China and ${ }^{3}$ Department of Physics, \\ Zunyi Normal College, Zunyi 563002, People's Republic of China \\ Email: \\ Hong Chen* - chenh@swu.edu.cn \\ * Corresponding author \\ Keywords: \\ current-perpendicular-to-plane geometry; Heusler alloy; \\ nonequilibrium Green's function; spin transport; spintronics; spin valve
}

Beilstein J. Nanotechnol. 2019, 10, 1658-1665. doi:10.3762/bjnano.10.161

Received: 19 January 2019

Accepted: 24 July 2019

Published: 08 August 2019

Associate Editor: J. M. van Ruitenbeek

(C) 2019 Feng et al.; licensee Beilstein-Institut. License and terms: see end of document.

\begin{abstract}
A $\mathrm{Ti}_{2} \mathrm{NiAl}$ inverse Heusler alloy based current-perpendicular-to-plane (CPP) spin valve (SV) with various kinds of atomic terminated interfaces has been designed to explore the potential application of Heusler alloys in spintronics devices. By performing first principles calculations combined with the nonequilibrium Green's function, it is revealed that spin magnetic moments of interfacial atoms suffer a decrease, and the electronic structure shows that the $\mathrm{TiNi}^{\mathrm{B}}$-terminated structure possesses the largest interface spin polarization of $\approx 55 \%$. Our study on spin-transport properties indicates that the total transmission coefficient at the Fermi level mainly comes from the contribution from the spin up electrons, which are regarded as the majority of the spin electrons. When the two electrodes of the CPP-SV device are in parallel magnetization configuration, the interface containing Ti and Ni atoms possesses a higher spin up transmission coefficient than the interface containing $\mathrm{Ti}$ and $\mathrm{Al}$ atoms. The device with the $\mathrm{TiNi}^{\mathrm{B}}$-terminated interface possesses the largest magnetoresistance ratio of $3.28 \times 10^{5}$, and it has great application potential in spintronics devices.
\end{abstract}

\section{Introduction}

Since the first theoretical prediction of the half metallicity of Heusler alloys [1], there has been explosive interest in manipulating the electron spin in a Heusler-alloy-based spintronic device [2-4]. As one of the most important spintronics devices, a current-perpendicular-to-plane (CPP) spin valve (SV) based on the giant magnetoresistive (GMR) effect consists of a non- magnetic metal as a spacer that is sandwiched between two ferromagnetic materials. It can produce two distinct states: a low-resistance state, when two electrodes are in parallel magnetization configuration, and a high-resistance state, when they are in antiparallel magnetization configuration. Half-metallic Heusler alloys (HMHAs) are regarded as one of the most prom- 
ising candidates for electrode materials in CPP-SV owing to their high Curie temperature, tunable electronic structure and small lattice mismatch with $\mathrm{Ag}$ or $\mathrm{Cu}$. Moreover, the majority spin bands of HMHA across the Fermi level show typical metallicity, while minority spin bands possess an energy gap around the Fermi level. Such a novel band structure results in a theoretical $100 \%$ spin polarization, which is one of the most crucial parameters for CPP-SV according to the Valet-Fert model [5]. As one of the subfamilies of Heusler alloys, conventional Heusler alloys with space group FM-3M have a chemical formula of $\mathrm{X}_{2} \mathrm{YZ}$ where the $\mathrm{X}$ atom locates at $(0,0,0)$ and $(0.5$, $0.5,0.5)$ sites, and the $\mathrm{Y}$ and $\mathrm{Z}$ atoms sit at $(0.25,0.25,0.25)$ and $(0.75,0.75,0.75)$ sites. From an experimental point of view, a superconducting spin-valve effect has been demonstrated in a $\mathrm{Co}_{2} \mathrm{Cr}_{1-x} \mathrm{Fe}_{x} \mathrm{Al}$-based spin valve [6]. A CPP-SV using $\mathrm{Co}_{2} \mathrm{Mn}\left(\mathrm{Ga}_{0.25} \mathrm{Ge}_{0.75}\right)$ has been verified to have a high resistance-area product $(\triangle R A)$ of $6.1 \mathrm{~m} \Omega \cdot \mu \mathrm{m}^{2}$ and magnetoresistance (MR) ratio of 40.2\% [7]. $\mathrm{A} \mathrm{Co}_{2} \mathrm{Fe}\left(\mathrm{Ge}_{0.5} \mathrm{Ga}_{0.5}\right)$ [8] based CPP-SV obtained a higher $\triangle R A$ of $26.4 \mathrm{~m} \Omega \cdot \mu \mathrm{m}^{2}$ and a MR ratio of $129.1 \%$ [9]. Several CPP-SVs have employed conventional Heusler alloys such as $\mathrm{Co}_{2} \mathrm{Fe}_{0.4} \mathrm{Mn}_{0.6} \mathrm{Si}[10]$ and $\mathrm{Co}_{2} \mathrm{MnSi}$ [11], also reaching a high MR ratio. On the other hand, a state-of-the-art theoretical approach that combines first principles calculations with the Keldysh nonequilibrium Green's function theory is also an effective way to study the transport properties of a device. A high MR ratio of $174 \%$ was reported in a recent work on $\mathrm{Fe}_{4} \mathrm{~N}$-based CPP-SV, and its spinpolarized quantum transport properties were investigated [12]. The CoFeMnSi-based heterostructure exhibited an ultrahigh tunnel magnetoresistance (TMR) ratio of $2 \times 10^{3}$ [13]. Nonequilibrium spin injection in a MnAl-based spintronics device was studied, and a TMR ratio of $2000 \%$ was predicted under a high bias voltage [14]. A large TMR ratio and spin Seebeck effect were found in a $\mathrm{Ti}_{2} \mathrm{MnAl}$-based heterostructure [15]. Although some relatively high MR ratios have been predicted in CPP-SV devices, there is more room for further improvement in the MR value by using better half-metallic Heusler compounds. In addition to conventional Heusler compounds, much attention has been paid to a new subfamily of Heusler compounds, i.e., inverse Heusler compounds which have a space group of $\mathrm{F}-43 \mathrm{M}$ and chemical formula of $\mathrm{X}_{2} \mathrm{YZ}$ where the $\mathrm{X}$ atom locates at $(0,0,0)$ and $(0.25,0.25,0.25)$ sites, and the $\mathrm{Y}$ and $\mathrm{Z}$ atoms sit at $(0.5,0.5,0.5)$ and $(0.75,0.75,0.75$ sites [16-18]. Half-metallicity has been predicted in $\mathrm{Sc}_{2} \mathrm{MnSi}$ [19], $\mathrm{Ti}_{2} \mathrm{RuSn}$ [20], and $\mathrm{Ti}_{2} \mathrm{NiAl}$ [21]. Spin gapless semiconductor characteristics are also demonstrated in $\mathrm{Mn}_{2} \mathrm{CoAl}$ [22-25] and $\mathrm{Ti}_{2} \mathrm{MnAl}$ $[26,27]$. The interface characteristics of heterostructures based on inverse Heusler alloys have been studied in detail [28-30] Therefore, inverse Heusler compounds exhibit exceptional electronic structure and magnetic properties, and they deserve to be further studied and applied in spintronics devices.
In this study, we built a CPP-SV device employing a halfmetallic inverse Heusler alloy $\mathrm{Ti}_{2} \mathrm{NiAl}$ as the electrode and $\mathrm{Ag}$ as the spacer. Different atomic-terminated interfaces are considered. We performed the first-principles density functional theory combined with nonequilibrium Green's function to investigate the interfacial electronic structure, magnetic properties and MR ratio of the device.

\section{Results and Discussion}

Our investigated device is a two-probe device, where $\mathrm{Ti}_{2} \mathrm{NiAl}$ is employed as a semi-infinite left and right electrode, and $\mathrm{Ag}$ is selected to be the middle spacer layer. For bulk $\mathrm{Ti}_{2} \mathrm{NiAl}$, one Ti atom locates at the $(0,0,0)$ site, which is described as $\mathrm{Ti}^{\mathrm{A}}$, and the other locates at the $(0.25,0.25,0.25)$ site, which is described as $\mathrm{Ti}^{\mathrm{B}}$, where $\mathrm{Ni}$ and $\mathrm{Al}$ sit at $(0.5,0.5,0.5)$ and $(0.75$, $0.75,0.75)$ sites. When $\mathrm{Ti}_{2} \mathrm{NiAl}$ is applied to the device, there are two ideal terminations along the $\left(\begin{array}{lll}1 & 0 & 0\end{array}\right)$ direction: TiNi and TiAl. The TiNi-terminated interface can be further categorized into two patterns: (i) a $\mathrm{TiNi}^{\mathrm{T}}$-terminated interface where interfacial Ti and Ni atoms sit on the top of Ag atoms (see Figure 1a), and (ii) a $\mathrm{TiNi}^{\mathrm{B}}$-terminated interface where interfacial $\mathrm{Ti}$ and $\mathrm{Ni}$ atoms locate in the bridge sites between $\mathrm{Ag}$ atoms (see Figure 1c). In a similar manner, the TiAl-terminated interface can be further categorized into $\mathrm{TiAl}^{\mathrm{T}}$ (see Figure $1 \mathrm{~b}$ ) and $\mathrm{TiAl}^{\mathrm{B}}$ (see Figure 1d) terminated interfaces.

In order to study the magnetic behavior of $\mathrm{Ti}_{2} \mathrm{NiAl} / \mathrm{Ag} / \mathrm{Ti}_{2} \mathrm{NiAl}$ CPP-SV, we calculated the spin-resolved atom magnetic moment of each layer of the device with various atomic-terminated interfaces, which are shown in Figure 2. It can be seen that the magnetic moment of interfacial $\mathrm{Ti}^{\mathrm{b}}$ atoms in the $\mathrm{TiAl}^{\mathrm{T}}$ terminated (see Figure $2 \mathrm{a}$ ) and the $\mathrm{TiAl}^{\mathrm{B}}$-terminated structure (see Figure 2b) suffer from reduction compared to its value in bulk $\mathrm{Ti}_{2} \mathrm{NiAl}$. Besides, magnetic moments of interfacial $\mathrm{Ti}^{\mathrm{a}}$ and interfacial $\mathrm{Ni}$ atoms in $\mathrm{TiNi}^{\mathrm{T}}$ terminated structures (see Figure 2c) also decrease, and they become lower in $\mathrm{TiNi}^{\mathrm{B}}$ terminated structures (see Figure 2d). This reveals that, for an interface containing $\mathrm{Ti}$ and $\mathrm{Ni}$ atoms, the hybridization between interfacial $\mathrm{Ti}$ and $\mathrm{Ni}$ atoms in the $\mathrm{TiNi}^{\mathrm{B}}$-terminated structure is stronger than those in the $\mathrm{TiNi}^{\mathrm{T}}$-terminated structure. The total interfacial magnetic moments of $\mathrm{TiAl}^{\mathrm{T}}$ and $\mathrm{TiAl}^{\mathrm{B}}$ terminated structures are $0.831 \mu_{\mathrm{B}}$ and $0.871 \mu_{\mathrm{B}}$, respectively. The TiNi ${ }^{\mathrm{T}}$-terminated structure has the highest total interfacial magnetic moment of $1.06 \mu_{\mathrm{B}}$, while the $\mathrm{TiNi}^{\mathrm{B}}$-terminated structure owns the lowest total interfacial magnetic moment of $0.81 \mu_{\mathrm{B}}$. In addition, when $\mathrm{Ti}^{\mathrm{a}}, \mathrm{Ti}^{\mathrm{b}}$ and $\mathrm{Ni}$ are in the deep layer of the heterostructure, their magnetic moments are close to the values in $\mathrm{Ti}_{2} \mathrm{NiAl}$ bulk, indicating that interfacial effects have a minor influence on the magnetic moment of deep-layer atoms. The magnetic property of Al atoms can be explained by the Ruderman-Kittel-Kasuya-Yosida (RKKY) indirect exchange 


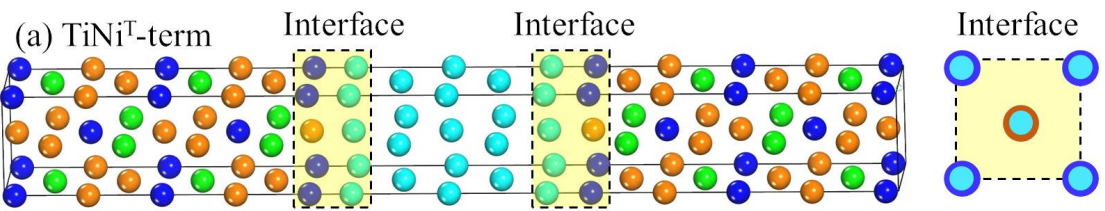

(b) $\mathrm{TiAl} l^{\mathrm{T}}$-term

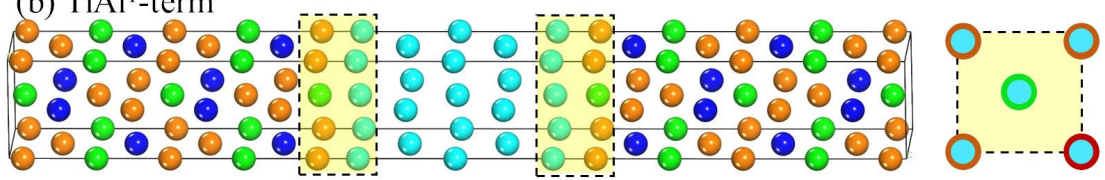

(c) $\mathrm{TiNi}^{\mathrm{B}}$-term

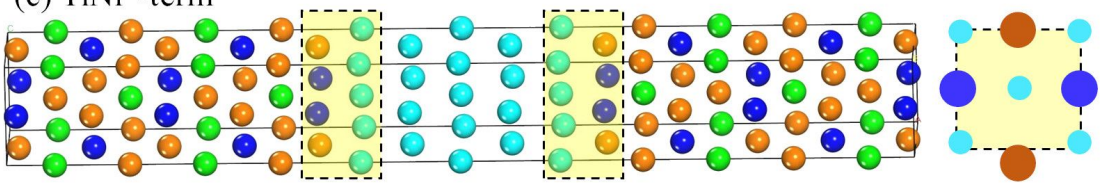

(d) $\mathrm{TiAl}^{\mathrm{B}}$-term

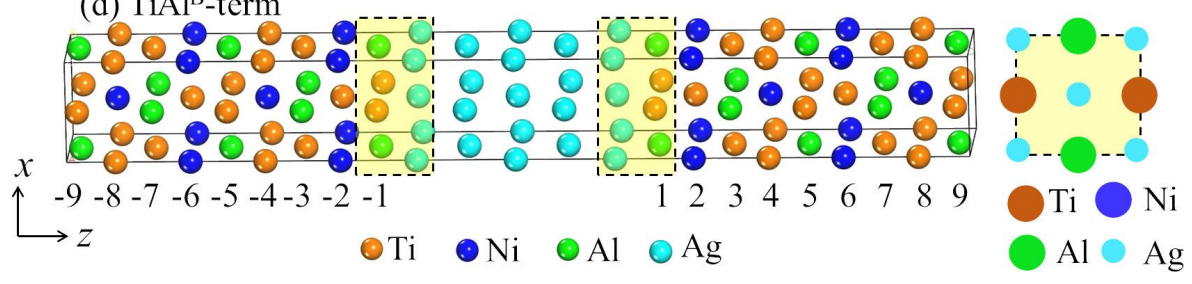

Figure 1: Schematic illustration of a $\mathrm{Ti}_{2} \mathrm{NiAl} / \mathrm{Ag} / \mathrm{Ti}_{2} \mathrm{NiAl}$ device with different atomic terminated interfaces. (a) $\mathrm{TiNi}^{\mathrm{T}}$-terminated interface, (b)TiAl ${ }^{\top}$ terminated interface, (c) $\mathrm{TiNi}^{\mathrm{B}}$-terminated interface and $(\mathrm{d}) \mathrm{TiAl}^{\mathrm{B}}$-terminated interface.

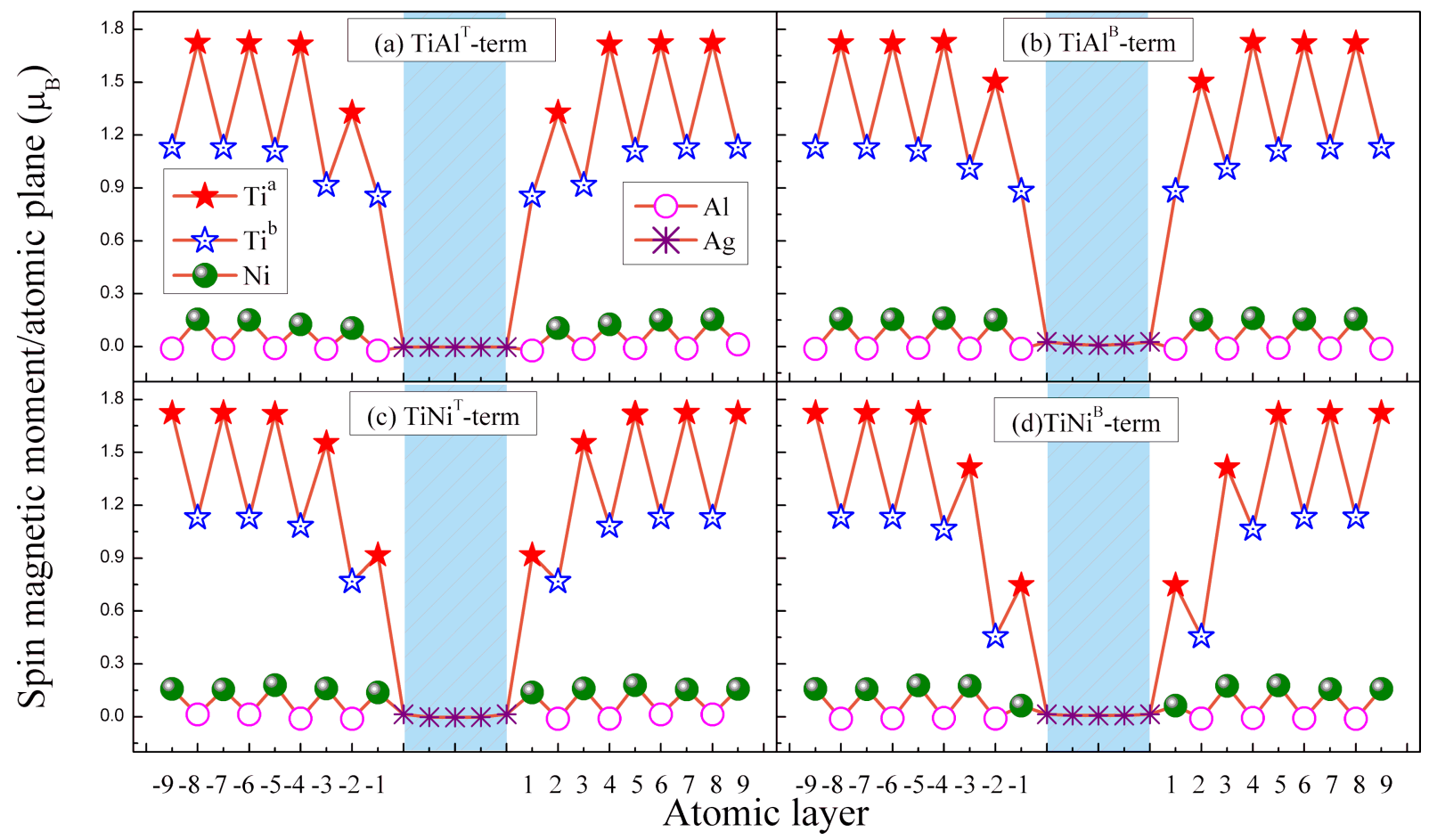

Figure 2: The calculated atomic spin magnetic moment of $\mathrm{Ti}_{2} \mathrm{NiAl} / \mathrm{Ag} / \mathrm{Ti}_{2} \mathrm{NiAl} \mathrm{CPP}-\mathrm{SV}$ at different atomic layers. (a) $\mathrm{TiAl}^{\mathrm{T}}$-terminated structure, (b) $\mathrm{TiAl}^{\mathrm{B}}$-terminated structure, (c) $\mathrm{TiNi}^{\mathrm{T}}$-terminated structure and (d) $\mathrm{TiNi}^{\mathrm{B}}$-terminated structure. 
mechanism. According to the RKKY mechanism, the magnetic coupling among d-electron atoms is transferred through the conduction electrons, and the cooperative magnetic states would exhibit ferromagnetic or antiferromagnetic alignment of the moments largely dependent upon the interatomic distances. Because the $\mathrm{Al}$ atom is a typical conduction sp-electron atom, it can continually exchange with local d-electrons of the nearest transition metal and serves as a bridge in hybridization between local d-electrons atoms. Hence, the atomic magnetic moment of the $\mathrm{Al}$ atom always presents a small negative value.

Because the interface spin polarization (ISP) plays an important role in determining the performance of a spin-dependent device, the interfacial electronic structure is calculated and exhibited in Figure 3, where the left panel and right panel indicate the interfacial electronic structure in spin up and spin down channels, respectively. It was observed that the spin down half- metallic energy gap in bulk $\mathrm{Ti}_{2} \mathrm{NiAl}$ is destroyed completely in various kinds of atomic-terminated interfaces of the $\mathrm{Ti}_{2} \mathrm{NiAl} /$ $\mathrm{Ag} / \mathrm{Ti}_{2} \mathrm{NiAl}$ device, which could be attributed to the appearance of interface states [27,28]. As for interfaces containing Ti and $\mathrm{Ni}$ atoms, spin up and spin down density of states at the Fermi level in the $\mathrm{TiNi}^{\mathrm{T}}$-terminated structure are close to those in the $\mathrm{TiNi}^{\mathrm{B}}$-terminated structure, and the spin up density of states at the Fermi level are higher than spin down density of states at the Fermi level. As for interfaces containing Ti and $\mathrm{Al}$ atoms, spin up and spin down density of states at the Fermi level in both $\mathrm{TiAl}^{\mathrm{T}}$ and $\mathrm{TiAl}^{\mathrm{B}}$ terminated structures are much lower than in $\mathrm{TiNi}^{\mathrm{T}}$ and $\mathrm{TiNi}^{\mathrm{B}}$ terminated structures, and the spin up density of states at the Fermi level are comparable to spin down density of states at the Fermi level. The ISP can be defined as ISP $=\left(N^{\uparrow}-N^{\downarrow}\right) /\left(N^{\uparrow}+N^{\downarrow}\right)$, where $N^{\uparrow}$ and $N^{\downarrow}$ represent the spin up and spin down contributions to the total density of states (DOS) at the Fermi level, respectively. Table 1

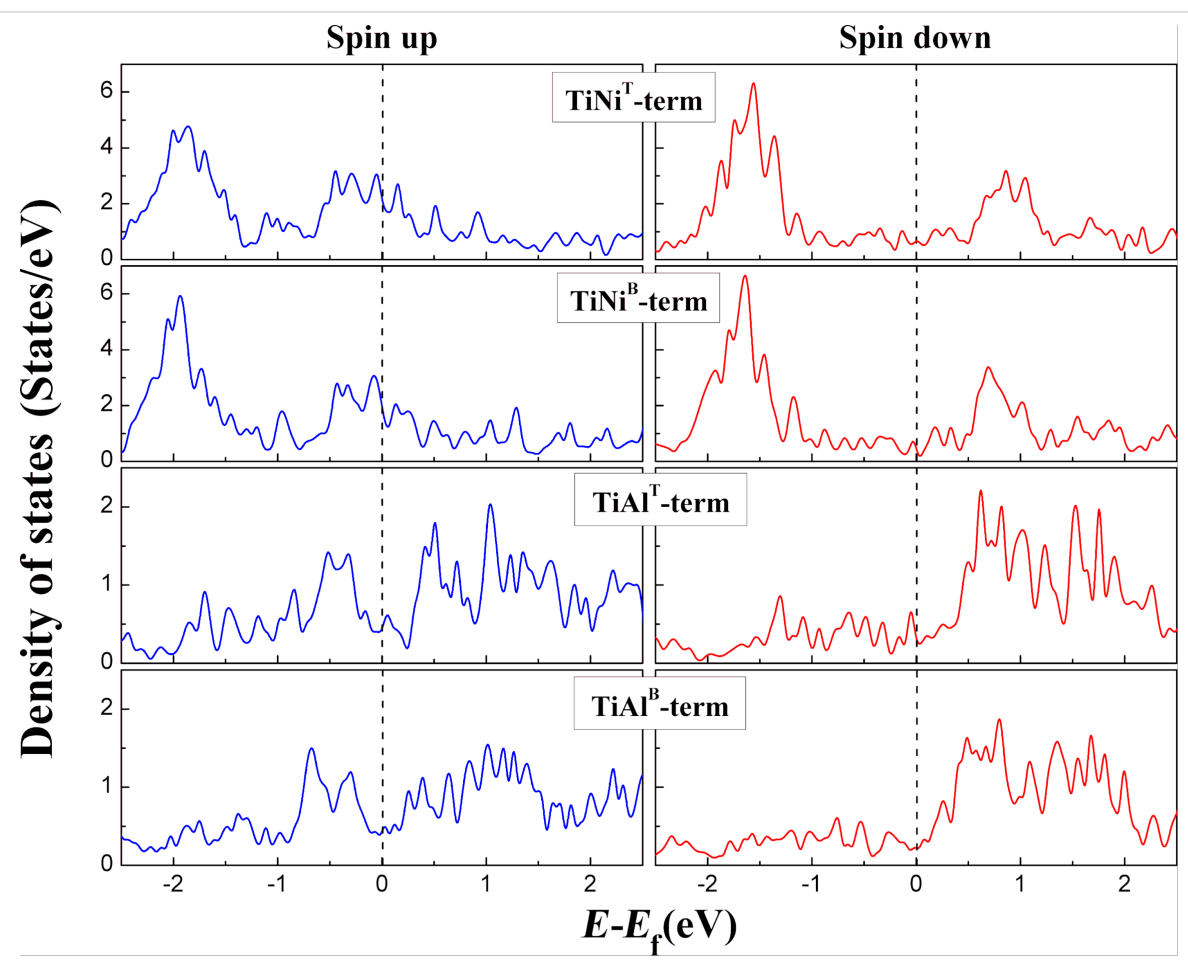

Figure 3: Density of states of the interface of the $\mathrm{Ti}_{2} \mathrm{NiAl} / \mathrm{Ag} / \mathrm{Ti}_{2} \mathrm{NiAl}$ device with different atomic terminated interface.

Table 1: The calculated interface spin polarization (ISP), transmission coefficients at the Fermi level, and magnetoresistance ratio (MR) of the Ti 2 NiAl/ $\mathrm{Ag} / \mathrm{Ti}_{2} \mathrm{NiAl}$ device with various atomic terminations.

\begin{tabular}{|c|c|c|c|c|c|c|}
\hline Termination & ISP & $T_{\mathrm{PC}}^{\mathrm{up}}\left(E_{\mathrm{f}}\right)$ & $T_{\mathrm{PC}}^{\text {down }}\left(E_{\mathrm{f}}\right)$ & $T_{\text {APC }}^{\text {up }}\left(E_{f}\right)$ & $T_{\mathrm{APC}}^{\text {down }}\left(E_{\mathrm{f}}\right)$ & MR \\
\hline $\mathrm{TiNi}^{\mathrm{T}}$ & $\approx 42 \%$ & 0.1067 & 0 & $0.1657 \times 10^{-6}$ & $0.1869 \times 10^{-6}$ & $3.03 \times 10^{5}$ \\
\hline $\mathrm{TiNi}^{\mathrm{B}}$ & $\approx 55 \%$ & 0.1152 & 0 & $0.2041 \times 10^{-6}$ & $0.1472 \times 10^{-6}$ & $3.28 \times 10^{5}$ \\
\hline $\mathrm{TiAl}^{\top}$ & $\approx 20 \%$ & 0.0408 & 0 & $0.2243 \times 10^{-6}$ & $0.1991 \times 10^{-6}$ & $9.64 \times 10^{4}$ \\
\hline $\mathrm{TiAl}^{\mathrm{B}}$ & $\approx 30 \%$ & 0.0991 & 0 & $0.7354 \times 10^{-6}$ & $0.7486 \times 10^{-6}$ & $6.67 \times 10^{4}$ \\
\hline
\end{tabular}


shows that the calculated ISP of the $\mathrm{TiAl}^{\mathrm{B}}$-terminated structure is $\approx 30 \%$, and it decreases to $\approx 20 \%$ in the $\mathrm{TiAl}^{\mathrm{T}}$-terminated structure, which is the lowest ISP value. However, the $\mathrm{TiNi}^{\mathrm{T}}$-terminated structure has a high ISP of $\approx 42 \%$, and the $\mathrm{TiNi}^{\mathrm{B}}$-terminated structure possesses the largest ISP of $\approx 55 \%$.

As for our two-probe device, the spin-dependent transmission coefficient $T^{\sigma}(E)$ can be calculated employing $T^{\sigma}(E)=\operatorname{Tr}\left[\Gamma_{\mathrm{L}} G^{\mathrm{R}} \Gamma_{\mathrm{R}} G^{\mathrm{A}}\right]$, where $\Gamma_{\mathrm{L}}$ and $\Gamma_{\mathrm{R}}$ are the coupling matrix of the left and right electrode, respectively; $G^{\mathrm{R}}$ and $G^{\mathrm{A}}$ are the retarded and advanced Green's function of the central region, respectively; and $\sigma$ is the spin direction, spin up or spin down. A two magnetization configuration is considered, thus the two electrodes of the device are in parallel magnetization configuration (PC) and in antiparallel magnetization configuration (APC). The spin-dependent electron transmission curves of spin up and spin down channels of the device with different atomic terminated interfaces in PC and APC are calculated and shown in Figure 4, where the green dashed line indicates the Fermi level, which is set to zero. It can be seen that in APC, for all four kinds of interface structures, both the spin up and spin down channels, possess a very small transport coefficient at the Fermi level. This indicates that the spin up and spin down channels are blocked when the device is in the APC state, i.e., the spin-polarized current cannot be detected, and the device is turned off. When the device is in the PC state, a spin up transmission coefficient at the Fermi level $\left(T_{\mathrm{PC}}^{\mathrm{up}}\left(E_{\mathrm{f}}\right)\right)$ is much higher than the spin down transmission coefficient at the Fermi level
$\left(T_{\mathrm{PC}}^{\text {down }}\left(E_{\mathrm{f}}\right)\right)$. This reveals that when the device is in the PC state, the spin up channel is unlocked and spin up electrons, which are regarded as the majority of spin electrons, can flow from the left electrode to the right electrode through a Ag spacer. In contrast, the spin down channel is still closed, and the spin down electrons, which are regarded as the minority of spin electrons, are suppressed. Hence, spin-polarized current can be detected when the device is in the PC state, and it is mainly dominated by spin up electrons, and the device is in a "turn on" mode. As for the device with an interface containing Ti and $\mathrm{Ni}$ atoms, in the PC state, the value of $T_{\mathrm{PC}}^{\mathrm{up}}\left(E_{\mathrm{f}}\right)$ in the $\mathrm{TiNi}^{\mathrm{T}}$ terminated structure $(0.1067)$ is comparable to the value in the $\mathrm{TiNi}^{\mathrm{B}}$-terminated structure (0.1152). In the APC state, the values of $T_{\mathrm{APC}}^{\mathrm{up}}\left(E_{\mathrm{f}}\right)$ and $T_{\mathrm{APC}}^{\mathrm{down}}\left(E_{\mathrm{f}}\right)$ in the $\mathrm{TiNi}^{\mathrm{T}}$-terminated structure are also close to the values in the $\mathrm{TiNi}^{\mathrm{B}}$-terminated structure. Besides, as for the device with an interface containing $\mathrm{Ti}$ and $\mathrm{Al}$ atoms, the value of $T_{\mathrm{PC}}^{\mathrm{up}}\left(E_{\mathrm{f}}\right)$ in the $\mathrm{TiAl}^{\mathrm{T}}$-terminated structure is 0.0408 , and it increases to 0.099 in the $\mathrm{TiAl}^{\mathrm{B}}$ terminated structure. Nevertheless, it can be found that the device with the TiNi-terminated interface possesses a higher spin up transmission coefficient than the TiAl-terminated interface.

In order to further exhibit the spin-transport behavior of the device, the transmission coefficient at the Fermi level of various kinds of structures in the two-dimensional Brillouin zone, which is perpendicular to the spin-transport direction, was calculated. In Figure 5, the contour plots of the transmission coefficient at the Fermi level are shown as a function of $k_{x}$ and

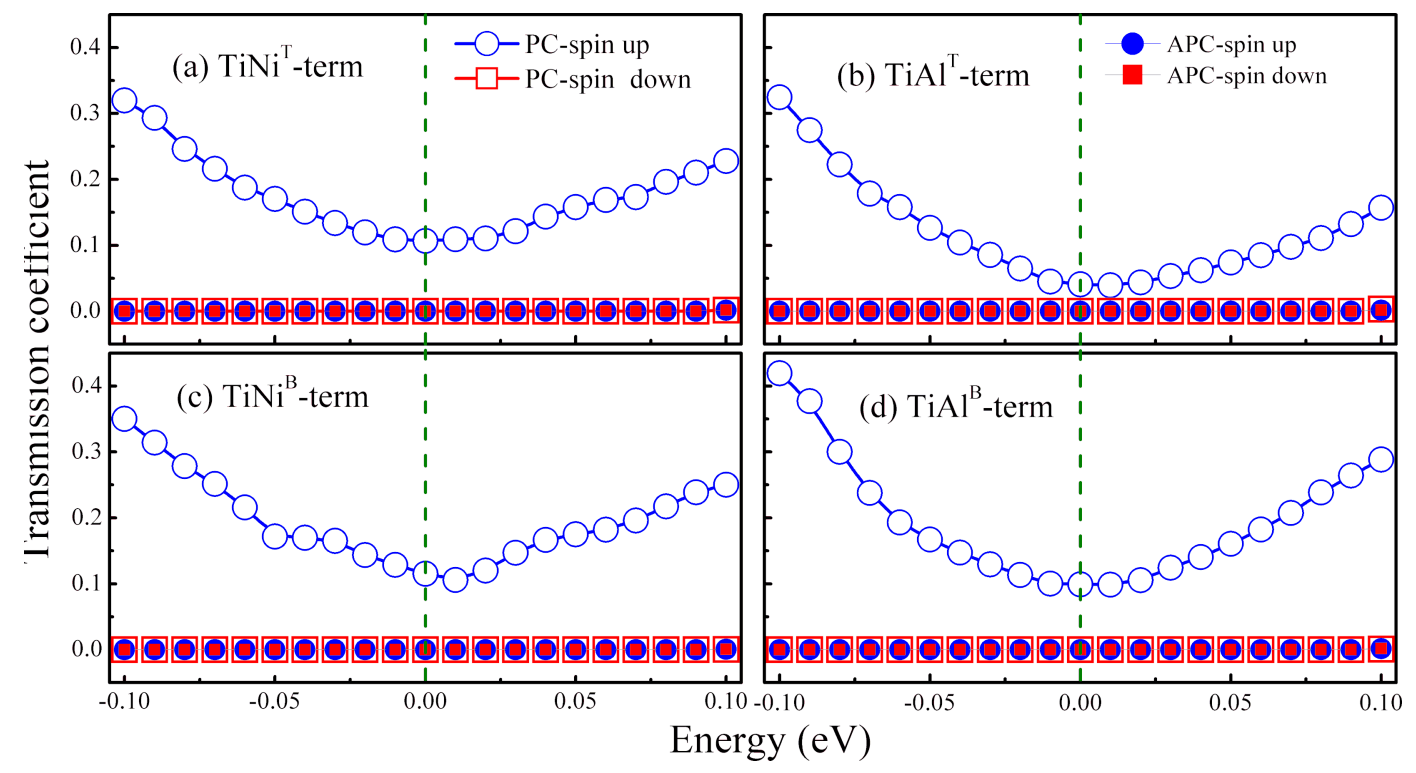

Figure 4: Transmission coefficient versus electron energy in the parallel magnetization configuration (PC) and antiparallel magnetization configuration (APC) of the $\mathrm{Ti}_{2} \mathrm{NiAl} / \mathrm{Ag} / \mathrm{Ti}_{2} \mathrm{NiAl}$ device with different atomic terminated interfaces. The dashed line is the Fermi level. (a) TiNi ${ }^{\mathrm{T}}$-terminated structure, (b) $\mathrm{TiAl}^{\mathrm{T}}$-terminated structure, (c) $\mathrm{TiNi}^{\mathrm{B}}$-terminated structure and (d) TiAl ${ }^{\mathrm{B}}$-terminated structure. 


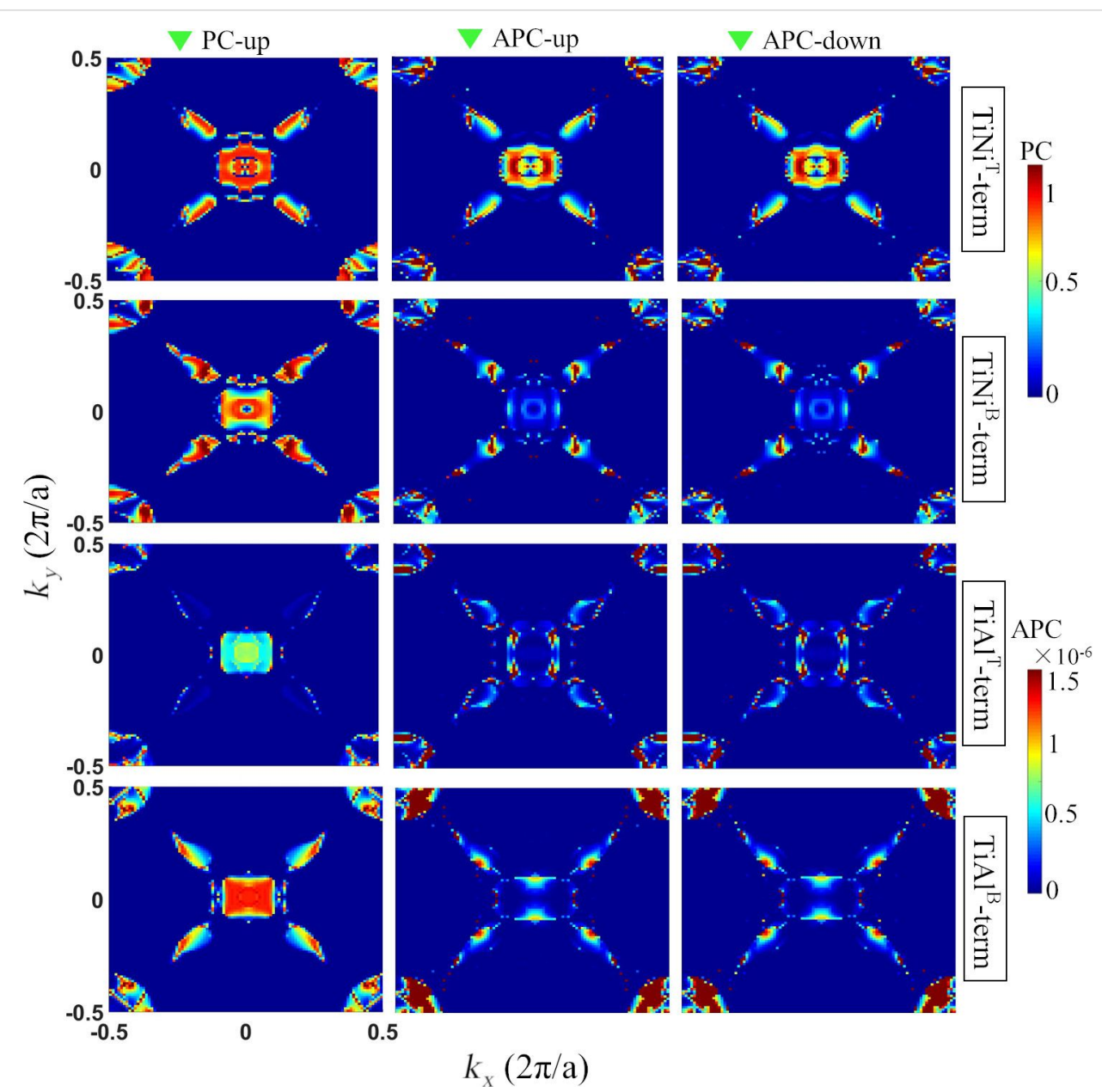

Figure 5: The $k_{/ / /}$-resolved transmission coefficients at the Fermi level of the $\mathrm{Ti}_{2} \mathrm{NiAl} / \mathrm{Ag} / \mathrm{Ti}_{2} \mathrm{NiAl} \mathrm{CPP}-\mathrm{SV}$ with different atomic terminated interfaces.

$k_{y}$ and indicate the transport behavior of spin up electrons of the device in the PC state. The plots in the middle column and the right column indicate the transport behavior of spin up and spin down electrons, respectively, of the device in the APC state. There are two color bars in Figure 5, and the upper color bar and the lower color bar represent the amplitude of the transport ability at various $\left(k_{x}, k_{y}\right)$ points when the device is in the $\mathrm{PC}$ and APC state, respectively. It was observed that when the device has different atomic terminated interfaces in the PC state, the magnitude of the hot spots in the spin up channel is much stronger than those in the spin up and spin down channels of the device in APC. This reveals that when the device is in the APC state, the transport ability of spin up and spin down electrons is inhibited, and spin-polarized electrons are less likely to travel from the left electrode to the right electrode. Besides, in the PC state the magnitude of the spin up channel of the $\mathrm{TiNi}^{\mathrm{T}}$-terminated structure is comparable to that of the $\mathrm{TiNi}^{\mathrm{B}}$ and $\mathrm{TiAl}^{\mathrm{B}}$ structures, while it becomes weaker in the $\mathrm{TiAl}^{\mathrm{T}}$ termination. This reveals that when the device in the PC state, the spin up electrons of the $\mathrm{TiNi}^{\mathrm{T}}, \mathrm{TiNi}^{\mathrm{B}}$ and $\mathrm{TiAl}^{\mathrm{B}}$ terminated structures have a similar intensity of transport ability, while the transport ability of the spin up electrons of the $\mathrm{TiAl}^{\mathrm{T}}$ terminated structure suffers from deterioration. Now, the situation when the device is in the APC state will be discussed. It can be seen that in the APC state, the transmission spectra of the spin up channel are nearly the same as that of the spin down channel for all kinds of atomic terminated structures, revealing that the transport ability of the spin up electrons is close to that of the spin down electrons. This results in the consequence that when the device is in the APC state, it is difficult to identify the spin up current and the spin down current from the total spin-polarized current.

As one the most significant parameters in spintronics devices, the magnetoresistance (MR) ratio (when the device at equilibrium) can be calculated by

$$
M R=\left|\frac{T_{\mathrm{PC}}\left(E_{\mathrm{f}}\right)-T_{\mathrm{APC}}\left(E_{\mathrm{f}}\right)}{\min \left(T_{\mathrm{PC}}\left(E_{\mathrm{f}}\right), T_{\mathrm{APC}}\left(E_{\mathrm{f}}\right)\right)}\right|,
$$


where $T_{\mathrm{PC}}\left(E_{\mathrm{f}}\right)$ and $T_{\mathrm{APC}}\left(E_{\mathrm{f}}\right)$ indicate the total transmission coefficient at the Fermi level in the PC and APC states, where

$$
\begin{aligned}
& T_{\mathrm{PC}}\left(E_{\mathrm{f}}\right)=T_{\mathrm{PC}}^{\text {up }}\left(E_{\mathrm{f}}\right)+T_{\mathrm{PC}}^{\text {down }}\left(E_{\mathrm{f}}\right), \text { and } \\
& T_{\mathrm{APC}}\left(E_{\mathrm{f}}\right)=T_{\mathrm{APC}}^{\text {up }}\left(E_{\mathrm{f}}\right)+T_{\mathrm{APC}}^{\text {down }}\left(E_{\mathrm{f}}\right) .
\end{aligned}
$$

The MR ratios of the $\mathrm{Ti}_{2} \mathrm{NiAl} / \mathrm{Ag} / \mathrm{Ti}_{2} \mathrm{NiAl}$ device with various kinds of atomic terminated interfaces were calculated and listed in Table 1. It can be seen that the MR ratios of all the structures exceed $\approx 10^{4}$ order of magnitude. The device with a TiAl ${ }^{\mathrm{B}}$ interface shows a high MR ratio of $6.67 \times 10^{4}$, and the MR ratio is boosted to a higher value of $9.64 \times 10^{4}$ for the $\mathrm{TiAl}^{\mathrm{T}}$ interface structure. This reveals that for the $\mathrm{Ti}_{2} \mathrm{NiAl} / \mathrm{Ag} / \mathrm{Ti}_{2} \mathrm{NiAl}$ device with an interface containing $\mathrm{Ti}$ and $\mathrm{Al}$ atoms, the Heusler layer sits on the top site of the $\mathrm{Ag}$ atom and can produce larger a MR ratio than when it sits at the bridge site of the $\mathrm{Ag}$ atom. On the other hand, the MR ratio of the device with the $\mathrm{TiNi}^{\mathrm{T}}$ terminated interface reaches up to $3.03 \times 10^{5}$, and the MR ratio is further enhanced to an ultrahigh value of $3.28 \times 10^{5}$ in the device with the $\mathrm{TiNi}^{\mathrm{B}}$ terminated interface. Therefore, it can be deduced that the $\mathrm{Ti}_{2} \mathrm{NiAl} / \mathrm{Ag} / \mathrm{Ti}_{2} \mathrm{NiAl}$ device with the interface containing $\mathrm{Ti}$ and $\mathrm{Ni}$ atoms generally results in a higher MR ratio than that with interfaces containing $\mathrm{Ti}$ and $\mathrm{Al}$ atoms. Additionally, the device with a $\mathrm{TiNi}^{\mathrm{B}}$-terminated interface possesses the largest MR value and can be regarded as a promising candidate for furture spintronics devices.

\section{Conclusion}

By employing first principles calculations combined with the nonequilibrium Green's function, we studied the interfacial magnetic properties, interfacial electronic structure and spin transport properties of a $\mathrm{Ti}_{2} \mathrm{NiAl} / \mathrm{Al} / \mathrm{Ti}_{2} \mathrm{NiAl} \mathrm{CPP}-\mathrm{SV}$ and four structures with different atomic terminated interfaces were modeled. Our calculation revealed that the magnetic moments of atoms located at the interface suffer a decrease, while the magnetic moments of atoms that sit at deep layers are close to corresponding values in bulk $\mathrm{Ti}_{2} \mathrm{NiAl}$. The $\mathrm{TiNi}^{\mathrm{T}}$-terminated structure possesses a high ISP of $\approx 42 \%$, and the $\mathrm{TiNi}^{\mathrm{B}}$-terminated structure has the largest ISP of $\approx 55 \%$. The total transmission coefficient at the Fermi level mainly comes from the contribution of the spin up electrons, which is regarded as the majority of spin electrons. In the PC state, the spin up transmission coefficients of $\mathrm{TiNi}^{\mathrm{T}}$ and $\mathrm{TiNi}^{\mathrm{B}}$ terminated structures are higher than that of $\mathrm{TiAl}^{\mathrm{T}}$ and $\mathrm{TiAl}^{\mathrm{B}}$ terminated structures. The MR ratios of the device with four different interfaces, i.e. $\mathrm{TiAl}^{\mathrm{T}}, \mathrm{TiAl}^{\mathrm{B}}, \mathrm{TiNi}^{\mathrm{T}}$ and $\mathrm{TiNi}^{\mathrm{B}}$ terminated interfaces, have been calculated. Our calculation reveals that the device with $\mathrm{TiNi}^{\mathrm{B}}-$ terminated structure possesses the largest MR ratio of $3.28 \times 10^{5}$, a value that is much higher than other CPP-SV devices such as $\mathrm{Fe}_{4} \mathrm{~N} / \mathrm{Ag} / \mathrm{Fe}_{4} \mathrm{~N}$ and $\mathrm{Co}_{2} \mathrm{MnAl} / \mathrm{Ag} / \mathrm{Co}_{2} \mathrm{MnAl}$.
Such a high MR ratio could be attributed to the complete spinpolarized $\mathrm{Ti}_{2} \mathrm{NiAl}$ bulk and high spin polarization at the interface of the device. Therefore, $\mathrm{Ti}_{2} \mathrm{NiAl} / \mathrm{Ag} / \mathrm{Ti}_{2} \mathrm{NiAl} \mathrm{CPP}-\mathrm{SV}$ has great application potential in spintronic devices.

\section{Simulation Details}

The $\mathrm{Ti}_{2} \mathrm{NiAl} / \mathrm{Ag} / \mathrm{Ti}_{2} \mathrm{NiAl}$ device with four different atomic terminated interfaces was geometrically optimized by utilizing a density functional theory (DFT)-based Vienna ab-initio simulation package (VASP) [31,32]. Ti $\left(3 d^{2} 4 s^{2}\right)$, Ni $\left(3 d^{8} 4 s^{2}\right)$, Al $\left(3 s^{2} 3 p^{1}\right)$ and $\mathrm{Ag}\left(4 d^{10} 5 s^{1}\right)$ were chosen to be the valence electron configurations. The exchange-correlation interaction is described by the Perdew-Burke-Ernzerhof (PBE) generalized gradient approximation (GGA). A Monkhorst-Pack grid of $13 \times 13 \times 1$ for $k$-point sampling, a self-consistent field (SCF) convergence criterion of $1 \times 10^{-5} \mathrm{eV}$, and a plane-wave basis cutoff energy of $550 \mathrm{eV}$ were applied. The Keldysh nonequilibrium Green's function (NEGF) theory, as implemented in Nanodcal package [33,34], was employed to investigate the spin-transport properties of the $\mathrm{Ti}_{2} \mathrm{NiAl} / \mathrm{Ag} / \mathrm{Ti}_{2} \mathrm{NiAl}$ device. In our calculations of transport properties, the number of Monkhorst-Pack k-space grids of the left and right electrode is $10 \times 10 \times 100$, and that of the central scattering region is $10 \times 10 \times 1$, where the self-consistent calculations are limited to $10^{-5}$ Hartree tolerance.

\section{Acknowledgements}

Y. F. acknowledges financial support from the National Natural Science Foundation of China (No. 11747114, 11875226 and 11874306).

\section{ORCID ${ }^{\circledR}$ iDs}

Hong Chen - https://orcid.org/0000-0003-4283-6095

\section{References}

1. de Groot, R. A.; Mueller, F. M.; Engen, P. G. v.; Buschow, K. H. J. Phys. Rev. Lett. 1983, 50, 2024-2027. doi:10.1103/physrevlett.50.2024

2. Hirohata, A.; Takanashi, K. J. Phys. D: Appl. Phys. 2014, 47, 193001. doi:10.1088/0022-3727/47/19/193001

3. Li, X.; Yang, J. Natl. Sci. Rev. 2016, 3, 365-381. doi:10.1093/nsr/nww026

4. Nakatani, T. M.; Furubayashi, T.; Kasai, S.; Sukegawa, H.; Takahashi, Y. K.; Mitani, S.; Hono, K. Appl. Phys. Lett. 2010, 96, 212501. doi:10.1063/1.3432070

5. Valet, T.; Fert, A. Phys. Rev. B 1993, 48, 7099-7113. doi:10.1103/physrevb.48.7099

6. Kamashev, A. A.; Validov, A. A.; Schumann, J.; Kataev, V.; Büchner, B.; Fominov, Y. V.; Garifullin, I. A. Beilstein J. Nanotechnol. 2018, 9, 1764-1769. doi:10.3762/bjnano.9.167

7. Takahashi, Y. K.; Hase, N.; Kodzuka, M.; Itoh, A.; Koganezawa, T.; Furubayashi, T.; Li, S.; Varaprasad, B. S. D. C. S.; Ohkubo, T.; Hono, K. J. Appl. Phys. 2013, 113, 223901. doi:10.1063/1.4809643 
8. Hase, N.; Varaprasad, B. S. D. C. S.; Nakatani, T. M.; Sukegawa, H.; Kasai, S.; Takahashi, Y. K.; Furubayashi, T.; Hono, K. J. Appl. Phys. 2010, 108, 093916. doi:10.1063/1.3503869

9. Takahashi, Y. K.; Srinivasan, A.; Varaprasad, B.; Rajanikanth, A.; Hase, N.; Nakatani, T. M.; Kasai, S.; Furubayashi, T.; Hono, K. Appl. Phys. Lett. 2011, 98, 152501. doi:10.1063/1.3576923

10. Sakuraba, Y.; Ueda, M.; Miura, Y.; Sato, K.; Bosu, S.; Saito, K.; Shirai, M.; Konno, T. J.; Takanashi, K. Appl. Phys. Lett. 2012, 101 , 252408. doi:10.1063/1.4772546

11. Sakuraba, Y.; Izumi, K.; Iwase, T.; Bosu, S.; Saito, K.; Takanashi, K.; Miura, Y.; Futatsukawa, K.; Abe, K.; Shirai, M. Phys. Rev. B 2010, 82, 094444. doi:10.1103/physrevb.82.094444

12. Feng, Y.; Cui, Z.; Wei, M.-s.; Wu, B. Appl. Surf. Sci. 2019, 466, 78-83. doi:10.1016/j.apsusc.2018.09.247

13. Han, J.; Feng, Y.; Yao, K.; Gao, G. Y. Appl. Phys. Lett. 2017, 111, 132402. doi:10.1063/1.4999288

14. Zhang, X.; Tao, L. L.; Zhang, J.; Liang, S. H.; Jiang, L.; Han, X. F. Appl. Phys. Lett. 2017, 110, 252403. doi:10.1063/1.4986449

15. Han, J.; Gao, G. Appl. Phys. Lett. 2018, 113, 102402. doi:10.1063/1.5047151

16. Skaftouros, S.; Özdoğan, K.; Şaşıoğlu, E.; Galanakis, I. Phys. Rev. B 2013, 87, 024420. doi:10.1103/physrevb.87.024420

17. Gilleßen, M.; Dronskowski, R. J. Comput. Chem. 2010, 31, 612. doi:10.1002/jcc.21358

18. Ma, J. H.; He, J. G.; Dipanjan, M.; Kamaram, M.; Sahar, K.; Tim, L.; Wolverton, C.; Ghosh, A. W.; Butler, W. H. Phys. Rev. B 2018, 98 , 094410. doi:10.1103/physrevb.98.094410

19. Ahmadian, F.; Salary, A. Intermetallics 2014, 46, 243-249. doi:10.1016/j.intermet.2013.11.021

20. Taşkın, F.; Atiş, M.; Canko, O.; Kervan, S.; Kervan, N. J. Magn. Magn. Mater. 2017, 426, 473. doi:10.1016/j.jmmm.2016.06.071

21. Lei, F.; Tang, C.; Wang, S.; He, W. J. Alloys Compd. 2011, 509, 5187-5189. doi:10.1016/j.jallcom.2011.02.002

22. Jakobsson, A.; Mavropoulos, P.; Şaşıoglu, E.; Blügel, S.; Ležaić, M.; Sanyal, B.; Galanakis, I. Phys. Rev. B 2015, 91, 174439. doi:10.1103/physrevb.91.174439

23. Ouardi, S.; Fecher, G. H.; Felser, C.; Kübler, J. Phys. Rev. Lett. 2013, 110, 100401. doi:10.1103/physrevlett.110.100401

24. Jamer, M. E.; Assaf, B. A.; Devakul, T.; Heiman, D. Appl. Phys. Lett. 2013, 103, 142403. doi:10.1063/1.4823601

25. Xu, G. Z.; Du, Y.; Zhang, X. M.; Zhang, H. G.; Liu, E. K.; Wang, W. H.; Wu, G. H. Appl. Phys. Lett. 2014, 104, 242408. doi:10.1063/1.4884203

26. Lukashev, P.; Kharel, P.; Gilbert, S.; Staten, B.; Hurley, N.; Fuglsby, R.; Huh, Y.; Valloppilly, S.; Zhang, W.; Yang, K.; Skomski, R.; Sellmyer, D. J. Appl. Phys. Lett. 2016, 108, 141901. doi:10.1063/1.4945600

27. Shi, W.; Muechler, L.; Manna, K.; Zhang, Y.; Koepernik, K.; Car, R.; van den Brink, J.; Felser, C.; Sun, Y. Phys. Rev. B 2018, 97, 060406. doi:10.1103/physrevb.97.060406

28. Li, J.; Jin, Y. Appl. Surf. Sci. 2013, 283, 876-880. doi:10.1016/j.apsusc.2013.07.036

29. Feng, Y.; Zhou, T.; Chen, X.; Yuan, H.; Chen, H. J. Phys. D: Appl. Phys. 2015, 48, 285302. doi:10.1088/0022-3727/48/28/285302

30. Feng, Y.; Wu, B.; Yuan, H.; Kuang, A.; Chen, H. J. Alloys Compd. 2013, 557, 202-208. doi:10.1016/j.jallcom.2012.12.134

31. Hohenberg, P.; Kohn, W. Phys. Rev. 1964, 136, B864-B871. doi:10.1103/physrev.136.b864
32. Kohn, W.; Sham, L. J. Phys. Rev. 1965, 140, A1133-A1138. doi:10.1103/physrev.140.a1133

33. Taylor, J.; Guo, H.; Wang, J. Phys. Rev. B 2001, 63, 245407. doi:10.1103/physrevb.63.245407

34. Waldron, D.; Haney, P.; Larade, B.; MacDonald, A.; Guo, H. Phys. Rev. Lett. 2006, 96, 166804. doi:10.1103/physrevlett.96.166804

\section{License and Terms}

This is an Open Access article under the terms of the Creative Commons Attribution License

(http://creativecommons.org/licenses/by/4.0). Please note that the reuse, redistribution and reproduction in particular requires that the authors and source are credited.

The license is subject to the Beilstein Journal of Nanotechnology terms and conditions:

(https://www.beilstein-journals.org/bjnano)

The definitive version of this article is the electronic one which can be found at: doi:10.3762/bjnano.10.161 